\title{
0 envelhecer na percepção de pessoas com pneumopatias crônicas submetidas à reabilitação pulmonar
}

\author{
Gabriela Matté Zanini", Carmen Célia Barradas Correia Bastos", \\ Joseane Rodrigues da Silva"**t, Keila Okuda Tavares ${ }^{* * *+}$
}

\section{Resumo}

O objetivo deste trabalho foi conhecer o que é envelhecer com uma doença pulmonar crônica, com base no olhar de indivíduos com pneumopatias crônicas, submetidos à reabilitação pulmonar. Trata-se de um estudo qualitativo, de caráter descritivo-exploratório. Foram realizadas entrevistas com portadores de doenças pulmonares crônicas submetidos a um programa de reabilitação pulmonar. A coleta de dados foi realizada por meio de entrevista semiestruturada, baseada na pergunta norteadora: "Para você, o que é envelhecer com doença pulmonar crônica?". Foram entrevistados sete indivíduos com idade média de 66 anos (50-71 anos). As entrevistas foram gravadas e transcritas na íntegra para serem analisadas com o método da análise do conteúdo.. Por meio da análise das falas, foi possível identificar três categorias: (1) a preocupação de envelhecer com uma doença limitante que não tem cura; (2) os sintomas que limitam o cotidiano e a vida social do sujeito com pneumopatia crônica; (3) a importância da adesão ao tratamento para o melhor envelhecimento. Os sintomas limitam suas atividades diárias gerando sentimentos negativos e isolamento social. As equipes de saúde devem compreender que envelhecer com doença limitante que não possui cura é uma experiência bastante complexa. É preciso investir tanto na assistência em saúde quanto na educação em saúde e estimular a formação de grupos de apoio formais e informais, para que os indivíduos acometidos tenham melhores condições físicas e emocionais para enfrentar a doença.

Palavras-chave: Fisioterapia. Envelhecimento. Doença crônica. Pneumopatias obstrutivas.

* Fisioterapeuta. Graduada pela Universidade Estadual do Oeste do Paraná (Unioeste).

** Pedagoga. Docente do Colegiado de Pedagogia da Universidade Estadual do Oeste do Paraná (Unioeste).

**** Fisioterapeuta. Docente do Colegiado de Fisioterapia da Universidade Estadual do Oeste do Paraná (Unioeste). Líder do Grupo de Pesquisa em Gerontologia da Unioeste.

***** Fisioterapeuta. Docente do Colegiado de Fisioterapia da Universidade Estadual do Oeste do Paraná (Unioeste). Membro do Grupo de Pesquisa em Gerontologia da Unioeste. Endereço para correspondência: Rua Paraná, 2447, CEP: 85812-011, Cascavel - PR. Telefone para contato: (45) 3220-3158. E-mail: keilaokudatavares@gmail.com.

$\rightarrow$ http://dx.doi.org/10.5335/rbceh.2013.3054 


\section{Introdução}

Com as mudanças demográficas e epidemiológicas verificadas atualmente, surgiram alterações na morbimortalidade da população, acarretando aumento significativo na incidência e surgimento de doenças crônicas (MARCON et al., 2005). Apesar de não causarem risco de vida imediato, as doenças crônicas, principalmente as respiratórias, provocam mudanças sociais, econômicas e individuais, levando os indivíduos acometidos a desenvolverem novos hábitos, modificando seus papéis sociais para incorporar a doença em seu viver (SILVA et al., 2005).

As doenças respiratórias crônicas afetam tanto as vias aéreas superiores quanto as inferiores, sendo a asma, a rinite alérgica e a doença pulmonar obstrutiva crônica (DPOC) as mais frequentes (BRASIL, 2010). Pessoas portadoras dessas doenças vivenciam situações limitantes por não conseguirem muitas vezes realizar as atividades de vida diária de forma satisfatória, bem como outras atividades relacionadas ao lazer e esportes. De todos os sintomas, a dispneia é o principal fator limitante, afetando diretamente a produtividade e a qualidade de vida. Todas essas modificações podem acarretar consequências negativas para a funcionalidade, como ansiedade e depressão, não afetando apenas a estrutura e funcionamento do organismo, mas também a autoestima $\mathrm{e}$ a percepção do indivíduo sobre si e seu envelhecer (CARVALHO et al., 2007; GODOY et al., 2009).

A percepção do envelhecer depende das implicações pessoais de cada indiví- duo, das possibilidades de adaptação às mudanças advindas do envelhecimento, de experiências, crenças e culturas próprias, entre outras condições intrínsecas associadas (TEIXEIRA; NERI, 2008). Quando os indivíduos deparam-se com uma situação de limitações e incapacidades e não existe o acompanhamento e o suporte necessários, essas condições tendem a se intensificar. Quanto maior o nível de dependência do indivíduo, maior será a negação perante si e sua condição, o que afetará o modo como se envelhece (TRENTINI et al., 2005; GODOY et al., 2009).

Os programas de reabilitação pulmonar apresentam nível 1 de evidência científica, consistindo em um tratamento que deve ser sempre indicado para indivíduos com pneumopatias crônicas, pelos seus resultados satisfatórios em relação aos ganhos na capacidade funcional, estado geral e qualidade de vida (DIRETRIZ DE REABILITAÇÃO CARDIOPULMONAR E METABÓLICA, 2006).

É fundamental na implantação desse tipo de programa levar em consideração que toda doença é uma experiência complexa e individualizada. Dessa maneira, os profissionais da saúde podem, por meio de uma abordagem que contemple as particularidades de cada indivíduo, visualizar o sujeito com pneumopatia como um todo e não apenas a sua doença, levando em consideração a multidimensionalidade do processo do envelhecer com uma doença crônica, para que, então, esses indivíduos sintam-se mais seguros e preparados para assumir seus papéis na sociedade de maneira o mais independente possível (RESENDE; NERI, 2009). 
Diante das limitações impostas pela doença pulmonar crônica, é importante compreender as percepções do envelhecer com essa doença partindo do olhar de pessoas com pneumopatias crônicas. Com essas informações, o atendimento dos profissionais da área da saúde pode se tornar mais humanizado e completo, visando não somente aos procedimentos técnicos em benefício do indivíduo acometido, mas também às necessidades e particularidades dessas pessoas envelhecentes, fatores importantes para o sucesso das abordagens das equipes de saúde.

Nesse sentido, o objetivo deste estudo é possibilitar a compreensão do que é envelhecer com uma doença pulmonar crônica por meio do olhar de pessoas com pneumopatias crônicas submetidas à reabilitação pulmonar.

\section{Materiais e métodos}

Trata-se de estudo qualitativo, de caráter descritivo-exploratório. Foram realizadas entrevistas, no período de agosto a outubro de 2011, com sete pacientes, de ambos os sexos, portadores de doenças pulmonares crônicas que estavam sendo tratados e acompanhados no Setor de Fisioterapia Respiratória do Centro de Reabilitação Física (CRF) da Universidade Estadual do Oeste do Paraná (Unioeste). Todos os indivíduos abordados são submetidos ao tratamento de reabilitação pulmonar há no mínimo um mês.

A coleta de dados foi realizada por entrevista semiestruturada, baseada na pergunta norteadora: "Para você, o que é envelhecer com doença pulmonar crônica?". Para realizar a coleta de dados, a pesquisadora responsável pelo estudo entrou em contato com os pacientes via telefone e, posteriormente à apresentação, forneceu esclarecimentos sobre a pesquisa, seus objetivos e sua forma de realização. Para aqueles que concordaram oralmente em participar, foi agendado um encontro em uma sala do CRF da Unioeste, onde os indivíduos entrevistados tinham privacidade para falar sobre suas vivências. Antes das entrevistas, todos leram e assinaram 0 Termo de Consentimento Livre e Esclarecido (TCLE).

As entrevistas foram gravadas utilizando-se gravador digital e transcritas na íntegra para análise posterior. Após cada entrevista, foram anotadas em um "diário de campo" as informações e impressões obtidas, com a finalidade de complementar os dados das entrevistas. Para melhor caracterizar os indivíduos entrevistados, foi aplicado um questionário com perguntas fechadas e abertas. Os textos transcritos foram identificados pela letra "E" de "entrevistado" e por números, sendo, em seguida, analisados com o método da análise do conteúdo (BARDIN, 2009).

A escolha da população estudada e a padronização da coleta de dados possibilitou que fossem cumpridas as regras da representatividade, exaustividade, homogeneidade e pertinência do material, propostas pelo método da análise do conteúdo. A análise dos dados obtidos permitiu o levantamento das hipóteses (afirmações provisórias) e indicadores para a análise e interpretação desses dados (BARDIN, 2009). 
Este estudo foi aprovado pelo Comitê de Ética em Pesquisa (CEP) da Unioeste sob o Parecer nº 324/2011.

\section{Resultados e discussão}

Durante o período de coleta de dados, foram atendidos 23 indivíduos no Setor de Fisioterapia Respiratória do CRF da Unioeste. Dentre esses, sete indivíduos apresentavam algum tipo de pneumopatia crônica. Tinham idade média de 66 anos (50 a 71 anos); 85,71\% apresentavam asma e $14,28 \%$ a DPOC. A maioria era do sexo feminino (85,71\%); sedentário até o início do atendimento fisioterapêutico $(71,42 \%)$; apresentavam história de tabagismo prévio por mais de 30 anos $(57,14 \%)$; tinham ensino fundamental incompleto $(85,71 \%)$; eram casados $(57,14 \%)$; e exerciam atividades relacionadas ao lar $(85,71 \%)$.

Os entrevistados relataram possuir dificuldade para realizar algum tipo de atividade no seu dia a dia e referiram, também, que já deixaram de realizar algum tipo de atividade devido à doença pulmonar crônica. Dentre as atividades de vida diárias mais limitantes, encontraram-se as atividades domésticas $(71,42 \%)$. Para a maioria dos entrevistados, o problema respiratório crônico os impedia de realizar a maioria das atividades que gostariam de fazer.

Após leitura exaustiva das falas e sua análise, foi possível identificar três categorias, descritas a seguir: (1) a preocupação de envelhecer com uma doença limitante que não tem cura; (2) os sintomas que limitam o cotidiano e a vida social da pessoa com pneumopatia crônica; (3) a importância da adesão ao tratamento para um melhor envelhecimento.

A preocupação de envelhecer com uma doença limitante que não tem cura

O sujeito com pneumopatia crônica visualiza de forma negativa o seu envelhecer pelo fato de o quadro clínico, além de limitante, ser crônico e irreversível. Foi possível observar que existe a preocupação com o futuro e o medo da morte "prematura", fatores que geram desgaste psicológico. Em relação ao futuro, esses indivíduos refletem sobre a possibilidade de piora dos sintomas e a situação de dependência que pode se desenvolver. É grande a preocupação do indivíduo acometido, por não querer se tornar dependente dos familiares ou não poder continuar cuidando da sua família.

Eu marco hora para morrer, envelhecer com essa doença (E1).

É um dilema. [...] a gente fica toda vida preocupada. Quando eu ficar mais velha, como é que vai ficar? [...] quanto mais velho a gente vai ficando, mais vai piorando (E3).

Estou cada vez ficando mais velha. Essa doença não vai mais sair de mim. Eu vou carregar para o resto da vida. Até o dia que for. [...] é penoso você carregar uma doença assim, junto com você. Você sabe que não tem cura [...]. É muito ruim. [...] vai prejudicando a saúde. Se é para aturar dois anos, atura um. Se é para aturar mais, cada vez menos. Se a pessoa não toma remédio, não faz a fisioterapia, [...] cada vez mais vai ficando pior (E7).

Eu acho difícil, porque eu sempre trabalhei desde criança, tenho meu filho pra cuidar e eu não queria ter uma doença assim (E6). 
Nos relatos, fica evidente o fato de que os indivíduos entrevistados não gostariam de estar envelhecendo da forma como estão. Gostariam de envelhecer sem a doença, o que, para eles, significaria envelhecer com mais saúde, independência e qualidade de vida.

Eu tenho que tomar muitos medicamentos, queria envelhecer assim, com uma vida saudável. [...] gostaria de ter uma vida saudável para eu ir lá correr, praticar um tipo de esporte. Fazer muita coisa que hoje, depois que eu estou doente, eu vi que não pude fazer. [...] eu não queria ter envelhecido desse tipo (E6).

Durante o processo de envelhecimento, são muitos os fatores que contribuem para que o indivíduo construa a percepção sobre o seu envelhecer, como as experiências individuais, sociais, ambientais, culturais, bem como fatores determinantes e modificadores da saúde. Por ser construído por meio das experiências únicas de cada sujeito, adquire significados diferentes dentro da sociedade (TEIXEIRA; NERI, 2008).

$\mathrm{O}$ envelhecimento é visto de forma negativa quando associado a alterações na saúde. No caso de indivíduos idosos que apresentam uma doença progressiva, limitante e sem cura, as dificuldades impostas pelo envelhecer tendem a se intensificar (TRENTINI et al., 2005), como verifica-se nos entrevistados deste estudo. Com a progressão da doença crônica e a piora do quadro clínico, o idoso vai alterando o modo como realiza o seu autocuidado e executa as suas atividades do cotidiano, levando-o à dependência em relação aos familiares, a qual, com o passar do tempo, aumenta cada vez mais (ALVES et al., 2007).
A dependência na terceira idade gera inversão de papéis sociais, pois, antes, quem cuidava passa a ser cuidado, o que acarreta alterações na dinâmica familiar. Na maioria das vezes, a dependência não é somente física, mas também financeira, pois o tratamento da doença crônica requer despesas contínuas. Nem sempre o Sistema Único de Saúde (SUS) arca com todos os custos, que passam, então, a ser suportados pelo orçamento familiar, podendo gerar alteração na estabilidade econômica da família (SILVA et al., 2002).

Nos casos em que o indivíduo com pneumopatia crônica para de trabalhar, principalmente aqueles que eram os provedores da renda familiar, surgem sentimentos de impotência, vergonha e, consequentemente, o isolamento social. Para esses sujeitos, o desligamento não está relacionado apenas ao espaço laboral e social, mas à sua essência, à unidade do ser humano em si. O indivíduo depara-se com o desligamento da atividade que, muitas vezes, dava sentido à sua vida e ajudava a construir sua personalidade (TRENTINI et al., 2005).

Os sintomas que limitam o cotidiano e a vida social da pessoa com pneumopatia crônica

Foi possível observar que os sintomas relacionados à doença pulmonar são fatores marcantes na vida dos indivíduos entrevistados. A tosse e a dispneia acabam dificultando e até mesmo impedindo que o indivíduo realize determinadas atividades da vida diária, como os cuidados com a higiene, vestuário, deambulação, afazeres domésticos e administração de medicamentos. 
Que nem o meu problema da tosse é bastante crítica, porque quando ela começa, até que ela não deu aquela volta que ela tem que dar, a tosse não para. Às vezes vão 15 minutos, até mais. [...] tu não consegue se virar direito, fazer uma coisa ou outra por causa do problema (E2).

É horrível, porque você fica privado de tudo o que você quer fazer. [...] falta $o$ ar, canseira, tosse e eu tinha muita tosse. Nossa, é horrível. [...] você não faz ideia como eu sofria pra sair na rua tossindo daquele jeito. Parecia uma louca. É horrível. [...] eu não podia fazer nada, eu largava tudo sabe, que era muita tosse, muita falta de ar, por qualquer movimento que você ia fazer para limpar a casa, varrer ou lavar roupa (E4).

Além das restrições relacionadas aos afazeres do dia a dia, existem ainda as limitações na vida social, as quais, muitas vezes, implicam o fato de que o indivíduo fica apreensivo ao sair de casa, com receio de que seus sintomas, como uma crise de tosse, afastem as pessoas de seu convívio, pois algumas sentem medo de ficar muito próximas por acharem que esse mal pode ser algo contagioso, o que resulta em constrangimento e, frequentemente, isolamento social.

Eu não podia nem sair na rua, dava vergonha quando eu saía na rua sabe, aquela tosse louca assim, que assombrava todo mundo, ninguém nem chegava perto da gente assim na rua, de tanta tosse. [...] eu sofria para sair na rua tossindo daquele jeito. Parecia uma louca! É horrível! (E4).

As doenças respiratórias crônicas representam um dos maiores problemas de saúde em nível mundial, sendo a asma e a DPOC as mais frequentes, com grande prevalência particularmente entre os idosos (BRASIL, 2010). Dentre os principais sintomas das doenças respiratórias crônicas, destacam-se a tosse e a dispneia. A tosse dificulta as relações sociais, pelo fato de algumas pessoas associarem-na com uma doença contagiosa, promovendo o preconceito e a discriminação social para com o indivíduo que a manifesta. Com frequência, o sujeito que apresenta tosse muito intensa, por receio ou vergonha, no intuito de evitar situações constrangedoras, prefere não interagir com outras pessoas (PINTO, 2005; TAVARES, 2009).

A dispneia é caracterizada por sensação subjetiva de falta de ar ou pela "respiração difícil", sendo o fator que mais dificulta as atividades de vida diária dos indivíduos com pneumopatia crônica (LANSING; GRACELY; BANZETT, 2009). É considerada limitante, pois o indivíduo diminui a realização de grande parte das atividades do seu dia a dia ou, até mesmo, deixa de executá-las. Passa a ser dependente de outras pessoas, principalmente, dos seus familiares, podendo surgir alterações emocionais como a ansiedade e a depressão, prejudicando ainda mais sua qualidade de vida (NOGUEIRA; LOPES, 2010; MARTÍNEZ-RIVERA et al., 2011).

O medo da intensificação dos sintomas e das limitações no dia a dia leva os idosos a refletirem constantemente sobre o seu futuro. Esse medo gera preocupação em relação à sua falta de capacidade para cuidar de si e da família, bem como uma perspectiva negativa em relação a um maior aproveitamento da vida em toda a sua plenitude, pela possibilidade de morte prematura. Tal situação acarreta desânimo e desmoti- 
vação para a manutenção do tratamento contínuo, que repercute no enfrentamento da doença e, consequentemente, gera grande desgaste psicológico, que, por sua vez, pode agravar ainda mais o quadro clínico da doença (PINTO, 2005; SILVA et al., 2005).

Para os idosos e, inclusive, indivíduos que estão envelhecendo com doença crônica, a luta por melhor qualidade de vida envolve a construção de uma nova forma de compreender a doença e a sua influência na situação atual, para assim conseguirem seguir uma vida mais próxima possível do "normal" com seus familiares e com a sociedade (RESENDE; NERI, 2009; SILVA et al., 2005).

A importância da adesão ao tratamento para um melhor envelhecimento

Os entrevistados relataram que para o "bom envelhecimento", o próprio indivíduo precisa se ajudar, aderindo ao tratamento, não somente ao medicamentoso, mas, principalmente, ao da reabilitação pulmonar, pois consideram que com esse tratamento passaram a ter melhor qualidade de vida. Os sintomas da doença como a tosse e a dispneia foram "amenizados" com o programa de exercícios e, como consequência, eles passaram a executar suas atividades de vida diária com menos dificuldade e a ter um melhor convívio familiar e social.

[...] é o tratamento que melhorou tudo. Que eu tive problema, tive falta de respiração, sofria sabe. Mas depois que eu comecei o tratamento, primeiro com o médico, depois com a reabilitação, hoje eu não sinto mais sufocado em ponto nenhum. Eu recuperei [...] eu diria $90 \%$. Estou respirando o suficiente, e hoje eu faço aquilo que eu quero fazer, eu sei os meus limites. Então, dentro do meu limite meu pulmão está ótimo. Graças a reabilitação. A reabilitação que me levantou (E5).

Para os entrevistados, é necessário aceitar e encarar a pneumopatia crônica, pois, dessa forma, ganham força para persistirem na luta diária do tratamento contínuo. É preciso conviver com a doença da melhor maneira possível, buscando adaptar o seu modo de ver a vida com a doença crônica.

A gente procura fazer o que pode para melhorar a vida, a saúde da gente [...]. Com doença ou sem doença o dia que Jesus chamar, nós estamos indo. $\mathrm{O}$ velho, o novo, chegou a hora, estamos indo(E3).

Os sintomas das pneumopatias crônicas podem ser amenizados com 0 tratamento adequado, tanto o medicamentoso, quanto o da reabilitação pulmonar (ZANCHET; VIEGAS; LIMA, 2005), mas, para isso, a adesão ao tratamento e a vontade do indivíduo em querer lutar por uma vida com mais qualidade é fundamental (TRENTINI et al., 2005; TEIXEIRA; NERI, 2008).

A reabilitação pulmonar é definida como um programa multidisciplinar, elaborado individualmente para o indivíduo com doença respiratória crônica. Conta com a atuação de profissionais da área da saúde, tais como os de Medicina, Fisioterapia, Enfermagem, Psicologia, Nutrição e Terapia Ocupacional. Tem como objetivo introduzir novamente a atividade física na vida do paciente, melhorando seu condicionamento físico, otimizando o desempenho físico, social e sua autonomia (CONSENSO BRASILEIRO SOBRE DOENÇA PULMONAR 
OBSTRUTIVA CRÔNICA, 2004; BRITISH THORACIC SOCIETY, 2001).

O programa de exercícios da reabilitação pulmonar reduz os sintomas, os níveis de ansiedade e depressão e as perdas funcionais. A melhora da tolerância ao exercício físico proporciona melhores condições para o desempenho das atividades de vida diária, gerando maiores níveis de independência e diminuição do isolamento social. A pessoa com pneumopatia crônica passa a ter melhor convívio com a família e a sociedade, o que repercute de forma positiva em sua qualidade de vida (ZANCHET; VIEGAS; LIMA, 2005; ROCETO et al., 2007).

É importante que o indivíduo acometido se conscientize e aceite sua condição. O processo de aceitação interfere diretamente em sua adesão ao tratamento (SILVA et al., 2005; PINTO, 2005), bem como na busca de outras estratégias de enfrentamento que auxiliem a ter mais força e disposição para encarar a doença de frente, a serem persistentes e determinados para alcançar o sucesso no tratamento e, assim, "vencer" a doença e conquistar um melhor envelhecer (TAVARES, 2009).

A busca de melhor qualidade da vida depende muito mais do indivíduo em si, de sua ânsia em querer melhorar do que de qualquer condição externa. $\mathrm{O}$ indivíduo deve perceber que a doença não precisa atuar somente como um fator debilitante, mas também como algo motivador, mobilizando a pessoa a superar seus medos e dificuldades (SILVA et al., 2005).

É de fundamental importância que os profissionais da saúde forneçam para esses sujeitos e seus familiares todo o apoio na área da educação e saúde. É necessária a implantação e estímulo da adesão a redes de apoio formal e informal, que visem a acompanhar o curso da doença, e, principalmente, é preciso ter a sensibilidade para perceber que esses indivíduos passam por situação não planejada em suas vidas, o que lhes causa medo e insegurança (TAVARES, 2009).

\section{Considerações finais}

Por meio da análise compreensiva das falas dos indivíduos que participaram dessa pesquisa, foi possível observar que esses apresentam preocupações em envelhecer com uma doença pulmonar crônica. Os sintomas limitam e muitas vezes impedem essas pessoas de realizarem suas atividades diárias, gerando sentimentos negativos relacionados à dependência, à morte prematura e, também, ao isolamento social.

A adesão ao tratamento é importante para a obtenção de melhor qualidade de vida. A reabilitação pulmonar apresenta papel fundamental na vida desses indivíduos, amenizando os sintomas, influenciando a retomada gradativa das suas atividades com menos dificuldades e maior independência.

Muitas vezes, o profissional da saúde preocupa-se com o tratamento da doença em si, acabando por esquecer que por trás dela existe uma pessoa com características e particularidades únicas, que também influenciam no processo saúde-doença, sendo tal compreensão fundamental para o sucesso do tratamento. $\mathrm{O}$ profissional deve ter o conhecimento de todos os aspectos que envolvem a vida 
do "ser" que está tratando, para que o atendimento torne-se mais completo, humanizado e individualizado, contribuindo assim para o sucesso da intervenção. ender que envelhecer com uma doença limitante que não possui cura é uma experiência bastante complexa. É preciso investir, tanto na parte da assistência quanto na educação em saúde e estimular a formação de grupos de apoio formais e informais, para que os indivíduos acometidos tenham melhores condições físicas e emocionais para enfrentar a doença.

Sugere-se, para estudos futuros, a abordagem do familiar que convive com os indivíduos que apresentam a pneumopatia crônica, pois são eles que acabam auxiliando o sujeito acometido a dar continuidade, no domicílio, ao tratamento orientado pelas equipes de saúde.
As equipes de saúde devem compre-

tent Analysis Method. Results: were interviewed seven individuals who had an average age of 66 years (50-71 years). Through the analysis of the speeches was possible to identify three categories: (1) the preoccupation of aging with a disease that has no cure, (2) the symptoms that limit daily and social life of people with chronic lung disease, (3) the importance of treatment adherence for better aging. Finals Considerations: symptoms limit daily activities generating negative feelings and social isolation. Health teams should understand that aging with a limiting disease is a complex experience. It is necessary invest both in health care and in health education and encourage the formation of support groups, formal and informal, so that the affected individuals have better physical and emotional conditions to face the disease.

Keywords: Physical therapy specialty. Aging. Chronic disease. Lung diseases, obstructive

\section{Referências}

\section{The aging in the perception of people with chronic lung diseases submitted to pulmonary rehabilitation}

\section{Abstract}

Objective: to know what is aging with a chronic lung disease through the view of people with chronic lung diseases undergoing Pulmonary Rehabilitation. Materials and Methods: this is a qualitative, descriptive and exploratory study. Interviews were conducted with persons with chronic lung disease who were undergoing a Pulmonary Rehabilitation Program. Data collection was performed using a semi-structured interview based on a guiding question: "For you, what is aging with chronic lung disease?". The interviews were recorded and transcribed for analysis with the Con-
ALVES, L. C. et al. Influência das doenças crônicas na capacidade funcional dos idosos do município de São Paulo, Brasil. Caderno de Saúde Pública, São Paulo, v. 23, n. 8, p. 1924-1930, 2007.

BARDIN, L. Análise de conteúdo. 5. ed. Lisboa-Portugal: Editora 70, 2009. 281 p.

BRASIL. Ministério da Saúde. Secretaria de Atenção à Saúde. Departamento de Atenção Básica. Doenças respiratórias crônicas/ Ministério da Saúde, Secretaria de Atenção à Saúde, Departamento de Atenção Básica. - Brasília: Ministério da Saúde, 2010. 160: il. - (Série A. Normas e Manuais Técnicos) (Cadernos de Atenção Básica, n. 25).

BRITISH THORACIC SOCIETY STATEMENT. London. Pulmonary Rehabilitation: British Thoracic Standards of Care Subcom- 
mitee on Pulmonary Rehabilitation. Thorax, v. 56, n. 1, p. 827-834, 2001.

CARVALHO, N. S. et al. Asma e doença pulmonar obstrutiva crônica: uma comparação entre variáveis de ansiedade e depressão. Jornal Brasileiro de Pneumologia, São Paulo, v. 33, n. 1, p. 1-6, 2007.

CONSENSO. II Consenso Brasileiro sobre Doença Pulmonar Obstrutiva Crônica (DPOC). Jornal Brasileiro de Pneumologia, São Paulo, v. 30, n. 5, p. 1-42, nov. 2004.

DIRETRIZ. Diretriz de reabilitação cardiopulmonar e metabólica: aspectos práticos e responsabilidades. Arquivos Brasileiros de Cardiologia, v. 86, n. 1, p. 74-82, 2006.

GODOY, R. F. et al. Repercussões tardias de um programa de reabilitação pulmonar sobre os índices de ansiedade, depressão, qualidade de vida e desempenho físico em portadores de DPOC. Jornal Brasileiro de Pneumologia, São Paulo, v. 35, n. 2, p. 129-136, 2009.

LANSING, R.; GRACELY, R.; BANZETT, R. The multiple dimensions of dyspnea: review and hypotheses. Respiratory Physiology \& Neurobiology, Amsterdam, v. 167, n. 1, p. 53-60, 2009.

MARCON, S. S. et al. Vivência e reflexões de um grupo de estudos junto às famílias que enfrentam a situação crônica de saúde. Revista Texto e Contexto Enfermagem, Florianópolis, v. 15, n. 14, p. 116-124, 2005.

MARTÍNEZ-RIVERA, C. et al. Psychological Profile of Patients with Bronchial Asthma and Functional Dyspnea: A Comparison with a Non-Asthmatic Population and Impact on the Disease. Archivos de Broncopneumologia, Barcelona, v. 47, n. 2, p. 73-78, 2011.

NOGUEIRA, K. T.; LOPES, C. Associação entre transtornos mentais comuns e qualidade de vida em adolescentes asmáticos. Revista Brasileira de Epidemiologia, São Paulo, v. 13, n. 3, p. 476-486, 2010.

PINTO, J. M. S. Vida sufocada: sofrimentos de pessoas com pneumopatias crônicas e a promoção da saúde pelo autocuidado. 2005. 161 f. Dissertação (Mestrado em Educação em Saúde) - Universidade de Fortaleza, Fortaleza, 2005.

RESENDE, M. C.; NERI, A. L. Ajustamento psicológico e perspectiva de velhice pessoal em adultos com deficiência física. Revista Psicologia em Estudo, Maringá, v. 14, n. 4, p. 767-776, 2009.

ROCETO, L. S. et al. Eficácia da reabilitação pulmonar uma vez na semana em portadores de doença pulmonar obstrutiva. Revista Brasileira de Fisioterapia, São Carlos (cidade), v. 11, n. 6, p. 475-480, 2007.

SILVA, D. G. V. et al. Qualidade de vida na perspectiva de pessoas com problemas respiratórios crônicos: a contribuição de um grupo de convivência. Revista Latino-americana de Enfermagem, Ribeirão Preto, v. 13, n. 1, p. 7-14, 2005.

SILVA, L. F. et al. Doença crônica: o enfrentamento pela família. Acta Paulista de Enfermagem, São Paulo, v. 15, n. 1, p. 40-47, 2002.

TAVARES, K. O. Fibrose cística: os múltiplos olhares para o ser fibrocístico. 2009. 140 f. Dissertação (Mestrado em Ciências da Saúde) - Universidade Estadual de Maringá, Maringá, 2009.

TEIXEIRA, I. N. D. O.; NERI, A. L. Envelhecimento bem-sucedido: uma meta no curso da vida. Revista de Psicologia USP, São Paulo, v. 19 , n. 1, p. 81-94, 2008.

TRENTINI, M. et al. Enfrentamento de situações adversas e favoráveis por pessoas idosas em condições crônicas de saúde. Revista Latino Americana de Enfermagem, Ribeirão Preto, v. 13, n. 1, p. 38-45, 2005.

ZANCHET, R. C.; VIEGAS, C. A. A.; LIMA, T. A eficácia da reabilitação pulmonar na capacidade de exercício, força da musculatura inspiratória e qualidade de vida de portadores de doença pulmonar obstrutiva crônica. Jornal Brasileiro de Pneumologia, São Paulo, v. 31, n. 2, p. 118-124, 2005. 